\title{
MICROENCAPSULATION TECHNIQUE FOR SELECTED PROBIOTICS AND PREBIOTICS
}

\author{
Ravi $\mathrm{D}^{\mathbf{1}^{*}}$, Usha $\mathrm{G}^{2}$, Parthasarathy $\mathrm{R}^{1}$ \\ ${ }^{1}$ Bioprocess unit, Department of Botany, Government Arts College, Coimbatore \\ ${ }^{2}$ Final year M.Tech. Biotechnology, Department of Biotechnology, Anna University, Regional centre, Coimbatore \\ *Corresponding author: dravi.botany@gmail.com
}

\begin{abstract}
Nowadays probiotics and prebiotics require full attention to stay viable in the food industry and the medical field. Microencapsulation is performed generally to give a protective covering for probiotics and prebiotics thereby saving from the spoilt environment. The Microencapsulation study is mainly performed in order to approve the viability of probiotics for better mode of action in food, health care and other applications. Prebiotics and sodium alginate was used as a coating material in order to microencapsulate probiotics (Lactobacillus rhamnosus and Lactobacillus plantarum). This research depicts the survival rate of encapsulated probiotics (L.plantarum and L.rhamnosus) with/without prebiotics over a period of one month.
\end{abstract}

(Key words: Microencapsulation, Probiotics, Prebiotics, Lactobacillus plantarum, Lactobacillus rhamnosus)

\section{INTRODUCTION}

\section{Encapsulation-The future of probiotics}

Entrapment of cells in a gel matrix of alginates is the most popular system of immobilization. The terms immobilization and encapsulation were used interchangeably in most reported literature. While encapsulation is the process of forming a continuous coating around an inner matrix that is wholly contained within the capsule wall as a core of encapsulated material, immobilization refers to the trapping of material within the capsule wall as a core of encapsulated material; immobilization refers to the trapping of material within or throughout a matrix. A small percentage of immobilized material may be exposed at the surface, while this is not the case for encapsulated material. Encapsulation occurs naturally when bacterial cells grow and produce Exopolysaccharides.

The microbial cells are entrapped within their own secretions that act as a protective structure or a capsule, reducing the permeability of the material through the capsule and therefore less exposed to adverse environmental factors. Many lactic acid bacteria synthesize Exo-polysaccharides, but they produce insufficient Exo-polysaccharides to be able to encapsulate themselves fully. Microencapsulation helps to separate a core material from its environment until it is released. It protects the unstable core from its environment, thereby improving its stability, extends the core's shelf life and provides a sustained and controlled release.

The structure formed by the micro-encapsulation agent around the core substance is known as the wall. The properties of the wall system are designed to protect the core and to release it at controlled rates under specific conditions while allowing small molecules to pass in and out of the membrane .The capsules may range from submicron to several millimeters in size and can be of different shapes.

\section{Microencapsulation}

Microencapsulation has many advantages. The microcapsule is composed of permeable, spherical and strong membranous wall. Therefore the bacterial cells are within the microcapsules. Sodium alginate is a food grade encapsulating agent which has been used to protect probiotic organisms during processing and storage. Sodium alginate, when combined with calcium chloride, forms beads consisting of calcium alginate gel matrix. There are several methods that can be used to produce beads including the emulsion and extrusion techniques [1]. Probiotic organisms can be released from the beads by sequestering calcium ions using sodium citrate or phosphate buffer. Encapsulation with calcium alginate has been found 
to improve the viability of probiotic organisms in frozen yoghurt during processing and storage [2]. Viability may be further improved by adjusting the concentration of sodium alginate up to $4 \%$ [3].

The improvement in viability of probiotic organisms is attributed to the structure of the sodium alginate gel, which permits the diffusion of nutrients while shielding the microorganisms from molecules that may cause stress [4]. Microencapsulation has been found to reduce probiotic cell death caused by oxygen toxicity [5]. Probiotic organisms have also been found to agglomerate in the centre of beads creating anaerobic regions. An additional layer can be applied to provide probiotic organisms with a barrier from molecules that may cause stress and oxygen.

An additional layer to calcium alginate beads can be achieved by applying a polymer coating such as chitosan or poly-L-lysine (PLL) to the beads. However, the coating may affect the overall size of the bead. Large beads are undesirable in yoghurt and many other products as they may detract from the mouth feel of the product. Beads with a diameter exceeding approximately $0.1 \mathrm{~mm}(100 \mu \mathrm{m})$ are detectable in the mouth. Therefore, beads ranging between $15 \mu \mathrm{m}$ and $100 \mu \mathrm{m}$ that do not compromise the viability of probiotic organisms during processing and storage are desirable.

\section{Freeze drying - prebiotics and probiotics}

Prebiotics are generally used to improve the viability of probiotic organisms during storage. It is essential that one million probiotic organisms per gram should be present in a product [4] in order to confer the health benefits of probiotic organisms. Shin and their research group [6] found that the viability of commercial Bifidobacterium spp in skim milk improved by $55.7 \%$ after 4 weeks of refrigerated storage when FOS was added. Prebiotics are also used to protect probiotic organisms during freeze drying as they provide extra solids.

Some strains of probiotic organisms are sensitive to freeze drying due to deterioration of physiological state of the cells. Cryoprotectants such as mannitol, sorbitol, sodium tripolyphosphate, xylitol, glycerol, raffinose, maltodextrin, erythritol, threitol, trehalose glucose and fructose play a role in improving the cold tolerance of the microorganisms.

Cold tolerance is improved as cryoprotectants accumulate within the cells and the osmotic difference with the external environment is reduced. Cryoprotectants added to a cell suspension also reduced injury and death. Cryoprotectants added to reduce the mortality of probiotic organisms in freezedried yoghurt effectively function by promoting the formation of extracellular amorphous ice glass instead of crystalline ice [7]. Amorphous ice glass is not a destructive upon the cell membrane of some probiotic organisms. The extent to which cryoprotectant is provided may vary between cultures.

\section{MATERIALS AND METHODS}

\section{Culture conditions}

Pure lyophilized culture of L. plantarum and L.rhamnosus were obtained from National Dairy Research Institute, Karnal. Bacterial strains were cultivated for 24 hours at $35^{\circ} \mathrm{C}$ in $5 \% \mathrm{CO} 2$ and $95 \%$ air atmosphere.

\section{Microencapsulation Of Synbiotics}

\section{Preparation of probiotic bacteria}

The culture was grown and propagated three times successively for activation at $37^{\circ} \mathrm{C}$ for 24 hours. Probiotic organisms were harvested by centrifuging at $10000 \mathrm{rpm}$ at $4^{\circ} \mathrm{C}$ for 20 minutes and the pellet was washed and suspended twice in saline solution. The final bacterial counts were adjusted to $10^{9} \mathrm{cells} / \mathrm{ml}$ and kept ready for encapsulation.

Intrusion method is the cheaper method and fastest method to encapsulate probiotics and prebiotics. Generally in intrusion method, Probiotic cultures were mixed with Alginate solution and fixed through a nozzle and injected into a hardening solution $\left(\mathrm{CaCl}_{2}\right)$ and allowed to stand for one hour for effective binding and encapsulation. Then, second coating solution of prebiotics were added and fixed through a nozzle and entrapped microcapsules forms spherical beads of synbiotics.

\section{Microencapsulation of Probiotics and Prebiotics}

Probiotic - Prebiotic microcapsules were prepared by mixing $1 \%(\mathrm{v} / \mathrm{v})$ of Lactobacillus plantarum and Lactobacillus rhamnosus and the previously autoclaved $\left(121^{\circ} \mathrm{C}, 15 \mathrm{~min}\right)$ prebiotics (fiber, $0 \%$ to $3 \%$ ). The extrusion technique of microencapsulation was used [8](Krasaeekoopt et al., 2004). After washing, $1 \%$ each of Lactobacillus plantarum and Lactobacillus rhamnosus was mixed with $50 \mathrm{ml}$ of 
$1 \%$ to $3 \%$ sodium alginate solution and sterilized at $121^{\circ} \mathrm{C}$ for 15 minutes. The cell suspension was injected through a $0.11 \mathrm{~mm}$ needle into sterile $0.1 \mathrm{M}$ $\mathrm{CaCl}_{2}$. The beads were approximately $0.5 \mathrm{~mm}$ in diameter and were allowed to stand for 1 hour for gelification, rinsed and kept at $4{ }^{\circ} \mathrm{C}$ for further use. Microencapsulated beads were then observed under Scanning electron microscope.

\section{Improvement Of Viability Of Probiotics}

\section{Freeze drying of synbiotic microcapsules slurry}

The freeze drying process for the encapsulated probiotics and prebiotics was performed using the freeze dryer (Lyodel, LYO1550, and India). An aqueous slurry of calcium alginate coated synbiotic microcapsules was transferred to $100 \mathrm{ml}$ round bottom flask and pre-frozen to $-40^{\circ} \mathrm{C}$ in the pre freezer bath at atmospheric pressure for $2 \mathrm{~h}$. It was allowed to freeze dry for 6 hours with reduction of pressure on the round bottom flask for $10^{-3} \mathrm{~m}$ bar and raising the shelf temperature of sample $+10^{\circ} \mathrm{C}$. After freeze drying, dry agglomerates of synbiotic microcapsules were obtained. The survival rate of the freeze dried probiotic, alginate and prebiotic encapsulated probiotic was estimated up to four weeks.

However, the synbiotic effects of probiotics and prebiotics are strain dependent. Only certain probiotics grow when supplemented with prebiotic carbon source. Yeo Siok et al reported different growth rates for 2 different strains of Lactobacillus with ten different prebiotics.

\section{Viability checks for encapsulated synbiotic}

The probiotic organisms were enumerated using the method prescribed by [9]Tharmaraj and Shah (2003). L.plantarum and L.rhamnosus were selectively enumerated on MRS agar using anaerobic incubation at $37^{\circ} \mathrm{C}$ for 72 hours. Encapsulated organisms were released from Calcium alginate using sodium citrate and phosphate buffer prior to enumeration. The combined viability of freeze dried L.plantarum and L.rhamnosus was enumerated using MRS agar[9] (Tharmaraj and Shah, 2003) by anaerobic incubation at $37^{\circ} \mathrm{C}$ for 48 hours.

\section{RESULT AND DISCUSSION}

\section{Freeze Dried Microcapsules}

The different combinations of prebiotic yielded various amounts of synbiotic microcapsules after freeze drying of the slurry. Higher composition of prebiotic incorporation during microencapsulation gave higher yield of synbiotic microcapsules. Initial probiotic count $\left(\mathrm{cfu} \mathrm{g}^{-1}\right.$ ) of freeze dried synbiotic microcapsule was varying. The viability of encapsulated probiotic cells, depend on the Physiochemical properties of the capsules. In fact, the type and the concentration of the coating material and initial cell numbers and bacterial strains are some basic parameters which are important to maintain the probiotic viability [10]. The viability of probiotic decreased with the increase in storage time (nearly after 3 to 4 weeks). Therefore, the mortality rate was different in the microcapsules. Difference in probiotic viability in different storage times was different in a specific type of synbiotic microcapsules.

\section{Scanning Electron Microscopic View Of Encapsulated Synbiotics}

\section{Properties of the microencapsulated bead}

The microstructures of the microcapsules were observed by scanning electron microscope (SEM). The probiotic microcapsules were in spherical shapes with group of groups of entrapped bacteria evident in the internal space and surrounded by the matrix. The probiotic microcapsules were formed using $3 \%$ sodium alginate which gave smooth surface and small pores. Raising the sodium alginate concentration increases the number of binding sites for $\mathrm{Ca}^{2+}$ ions. Therefore a denser cross linked gel structure was formed. The average size of the microparticles obtained after freeze drying was 10 $\mu \mathrm{m}$. In general, the morphological analysis of freeze dried microparticles showed spherical shape. However, the surface of the microparticles was wrinkled due to the encapsulated cells within the particles due to the encapsulated cells within the particles and loss of water content during the freeze drying process (Figure 1). 

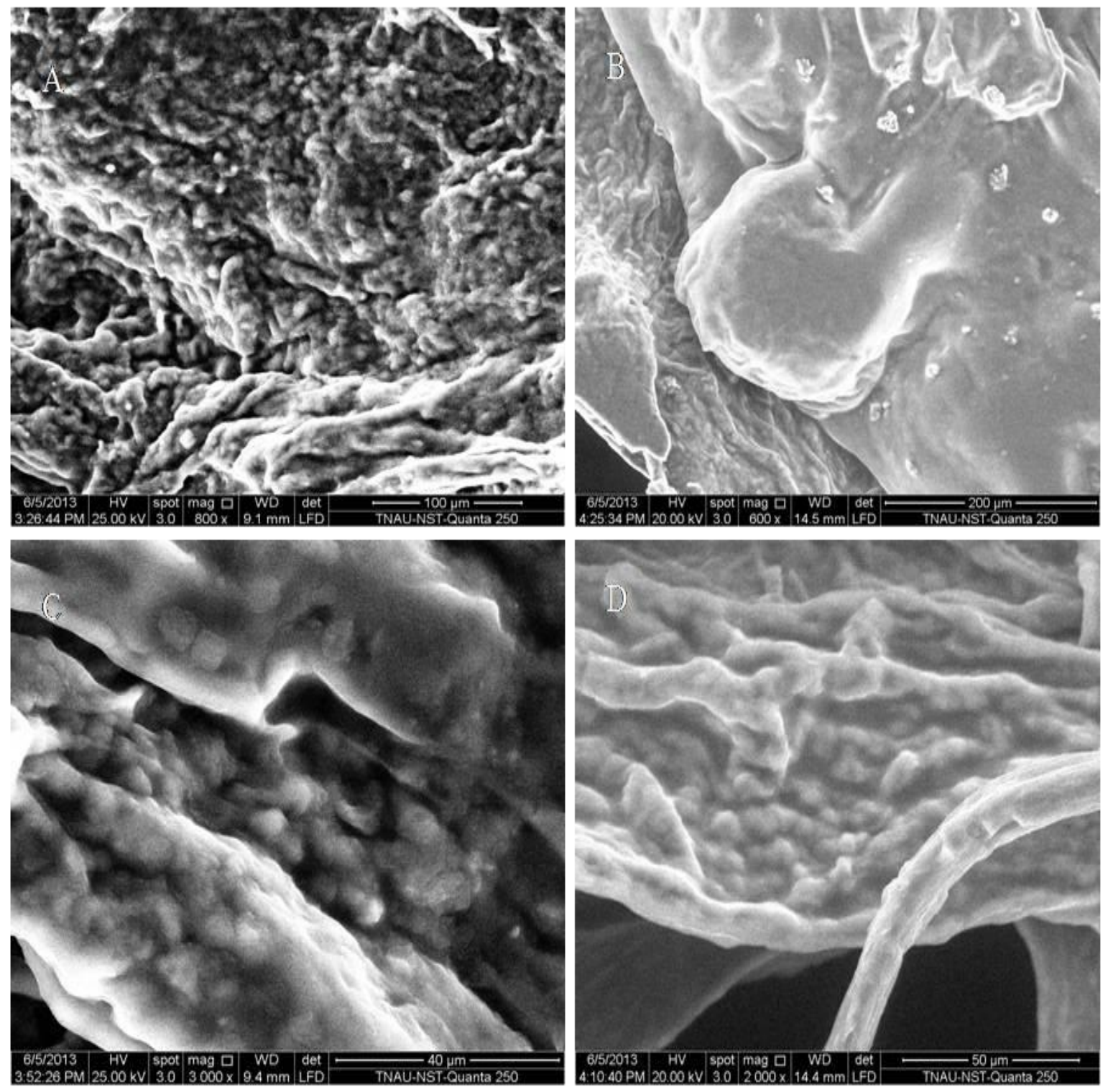

Figure 3.2.1 SEM image of Microencapsulated probiotic-prebiotic bead obtained by extrusion technique. A: Control (Lactobacillus plantarum), B: Lactobacillus plantarum coated with Borassus flabellifer and Sodium alginate, C: Control (Lactobacillus rhamnosus), D: Lactobacillus rhamnosus coated with Borassus flabellifer and Sodium alginate.

The Complex microstructure of the prebiotic-Caalginate microparticles loaded with L.plantarum and L.rhamnosus was obtained. The inner part of the microparticles was built of a mesh-like alginate network through which the bacterial groups were distributed and sequestered in voids. When compared the viability of L.plantarum and L.rhamnosus alone 
or with alginate, prebiotics, increased survival in the microparticles for $\log 4$ was observed.

The results indicated that microencapsulation of L.plantarum and L.rhamnosus using alginate as encapsulating material, prebiotics and calcium chloride L. rhamnosus followed by freeze drying offered significant protection of probiotic cells.

\section{Effect of freeze-drying on viability of encapsulated} synbiotics

The viability of microencapsulated L.plantarum, L.rhamnosus after freeze drying, stored at $4{ }^{\circ} \mathrm{C}$ for

Table 1: Survival rate of encapsulated probiotics (L.plantarum) with/without prebiotics over a period of 4 weeks

\begin{tabular}{|l|c|c|}
\hline \multicolumn{1}{|c|}{ Probiotic cultures, $(\log$ CFU/g) } & Stored 0 weeks & Stored 4 weeks \\
\hline L.plantarum+ alginate+ prebiotics & & \pm 8.56 \\
\hline L.plantarum + alginate & \pm 8.23 & \pm 5.98 \\
\hline
\end{tabular}

Table 2: Survival rate of encapsulated probiotics (L.rhamnosus) with/without prebiotics over a period of 4 weeks

\begin{tabular}{|l|c|c|}
\hline \multicolumn{1}{|c|}{ Probiotic cultures, $(\log$ CFU/g) } & Stored 0 weeks & Stored 4 weeks \\
\hline L.rhamnosus +alginate+ prebiotics & \pm 8.58 & \pm 7.41 \\
\hline L.rhamnosus+ alginate & \pm 8.59 & \pm 6.18 \\
\hline
\end{tabular}

for carrying out this kind of effective research in

four weeks is shown in Table -1 , Table -2 . The Government arts college, Coimbatore.

viability of Probiotic (L.plantarum) coated with prebiotics and sodium alginate showed highest (8.56 $\left.\log _{10} \mathrm{CFU} / \mathrm{g}\right)$ after encapsulated and $\left(5.98 \log _{10}\right.$ CFU/g) after storing for 4 weeks. The viability of Probiotic (L.rhamnosus) coated with prebiotics and sodium alginate showed highest (8.58 $\left.\log _{10} \mathrm{CFU} / \mathrm{g}\right)$ after encapsulated and (6.18 $\log _{10}$ $\mathrm{CFU} / \mathrm{g}$ ) after storing for 4 weeks. Also researchers [11] found that a storage temperature of $4^{\circ} \mathrm{C}$ was effective in maintaining the viability of microencapsulated synbiotics during storage.

\section{CONCLUSION}

Microencapsulation of synbiotics thus provides a major positive outcome in food biotechnology and in medical microbiology. Future research could be concentrated on improving the viability of probiotics with further stronger coating material for encapsulation.

\section{ACKNOWLEDGEMENT}

The author and Co-authors have a great sense of gratitude to UGC, Government of India, New Delhi 
5. Talwalkar A., and Kailasapathy K. (2003). 'Effect of microencapsulation on oxygen toxicity in probiotic bacteria', Aust. J. Dairy Technol, Vol 58, pp. 36-39.

6. Shin, H.S., Lee, J.H., Pestka, J.J., and Ustunol, Z. (2000). 'Viability of bifidobacteria in commercial dairy products during refrigerated storage', J. Food Prot, Vol 63, pp.327-331.

7. Shah, N. P. and Ravula, R., (2001)Microencapsulation of probiotic bacteria and their survival in frozen fermented dairy desserts, Aust. J. Dairy Technol., 55, 139-144.

8. Krasaekoopt W., Bhandari B., Deeth. H., (2003). 'Evaluation of encapsulation techniques of probiotics for yoghurt: a review', Int Diary J,Vol 13, pp. 3-13
9. Tharmaraj, N., Shah, N.P., (2003). Selective enumeration of Lactobacillus delbrueckii ssp. bulgaricus, Streptococcus thermophilus, Lactobacillus acidophilus, Bifidobacteria, Lactobacillus casei, Lactobacillus rhamnosus, and Propionibacteria. Journal of Dairy Science 86, 2288-2296.

10. Chen KN, Chen MJ, Liu JR, Lin CW, Chin HY. (2005); Optimization of incorporated prebiotics as coating materials for probiotic microencapsulation. J Food Sci. 70: M260M266.

11. Yeo SK, Liong MT. . (2010); Effect of prebiotics on viability and growth characteristics of probiotics in soymilkJ Sci Food Agric. 90:267-275. 